\title{
For All they that Take the Sword Shall Perish with the Sword-on the Radiation Hazard of The Use of Shells Containing Depleted Uranium
}

\author{
Okunev VS* \\ Bauman Moscow State Technical University, Russia \\ *Corresponding author: Okunev VS, Bauman Moscow State Technical University, 105005, 2nd Baumanskaya street, 5 Moscow, Russia. \\ To Cite This Article: Okunev VS, For All they that Take the Sword Shall Perish with the Sword-on the Radiation Hazard of The Use of Shells \\ Containing Depleted Uranium. Am J Biomed Sci \& Res. 2020 - 7(1). AJBSR.MS.ID.001107. DOI: 10.34297/AJBSR.2020.07.001107.
}

Received: 齿 September 24, 2019; Published: 眥 January 22, 2020

\begin{abstract}
Uranium is characterized by low activity. It is generally accepted that the biological (mainly carcinogenic) danger of using shells containing depleted uranium is associated with the toxicity of uranium dust. However, when a projectile collides with an object at high speed (1.6km/s or more), the half-life of uranium nuclei decreases significantly. In this case, the main biohazard factor is associated with radiation exposure.
\end{abstract}

Keywords: Uranium core shells, Transuranic elements, Electromagnetic interaction, Protons

\section{Introduction}

After the use by NATO forces of depleted uranium core shells in Iraq and Yugoslavia in the media, a discussion arose about possible radioactive contamination of the area and foci of cancer. Natural uranium isotopes are classified as long-lived nuclides. They are not dangerous under normal conditions. Uranium dust generated by a collision of an armor-piercing projectile with a tank armor or reinforced concrete object is toxic.

This can contribute to the formation of cancerous tumors. Uranium accumulates in human organs (in the liver, spleen, kidneys). The World Health Organization "accepts" soluble uranium salts for ordinary citizens at a level of $0.5 \mu \mathrm{g} / \mathrm{kg}$ body weight or $35 \mu \mathrm{g}$ for an adult weighing $70 \mathrm{~kg}$. The greatest danger is a significant (by many orders of magnitude) decrease in the half-life of uranium nuclei (including with respect to spontaneous fission) in the event of an armor-piercing projectile colliding with an object.

\section{Background-Known Facts}

The composition of natural uranium includes the isotopes $238 \mathrm{U}$ (99.28\%), 235U (0.72\%) and 234U (0.006\%). Depleted uranium is $238 \mathrm{U}$ with small impurities (usually less than $0.44 \%$ ) of $235 \mathrm{U}$. For $238 \mathrm{U}$, the half-life with respect to spontaneous fission is $~ 1016$ 1017 years, for 235U-1014-1015 years, for 234U-1012-1013 years.
In the absence of external influences, uranium (neither depleted nor enriched) does not constitute a radiation hazard.

Generally, nuclei are deformed upon transition to an excited energy state. The transition from the ground energy state to the excited energy state leads to a change in the geometric shape of the atomic nucleus. As a result, the core becomes less stable. Excitation is removed by spontaneous emission of $\gamma$-quanta, nucleons, lighter nuclei. Uranium-238 in an excited state is quickly divided. So, deformation of the shape of the nucleus in relation to the main energy state can lead to a significant decrease in half-life.

In 1962, the phenomenon of dividing isomerism was discovered. Isotopes of uranium and some transuranic elements $\mathrm{Pu}, \mathrm{Am}, \mathrm{Cm}$ and $\mathrm{Bk}$ ) have excited states with energies of about $2-3 \mathrm{MeV}$. Nuclei in such excited states spontaneously divide. The fission isomerism is explained by the difference in the shape of the nuclei in the ground and isomeric states. In experimental studies of Flerov and Petrzhak (1962) on nuclear isomerism, the half-life of uranium-238 via the spontaneous fission channel decreased to $0.3 \times 10-6 \mathrm{~s}$ [1].

Uranium fission when a projectile collides with an object the radius of the nuclear interaction does not exceed $\mathrm{rS} \sim 10-14 \mathrm{~m}$. The radius of the electromagnetic interaction is infinite. When atomic 
nuclei come together in two metallic colliding objects (a projectile and an obstacle) at distances exceeding $\mathrm{rS}$, the proton radius (size) of the nuclei is deformed as a result of electromagnetic interaction (mutual Coulomb repulsion of protons in the composition of nuclei). Protons in the atomic nucleus carry neutrons along and the nucleus is deformed. In this case, the geometric shape of the nucleus corresponds to the excited state. The core is divided $[2,3]$.

The article [2] provides estimates of the activity of the area when using shells containing depleted uranium. The specific activity is from $1.10 \times 106$ to $5.23 \times 1019 \mathrm{~Bq} / \mathrm{cm} 3$. Nuclear energy wastes characterized by specific activity from 111 to $3.7 \times 106 \mathrm{~Bq} /$ $\mathrm{cm} 3$ are classified as medium-active, and wastes with activity greater than $3.7 \times 106 \mathrm{~Bq} / \mathrm{cm} 3$ are highly active. Within a radius of $150 \mathrm{~m}$ from the place where the projectile collides with the object, an area contaminated with highly active waste is observed. In fission of uranium, neutrons are emitted (on average, with an energy of $2 \mathrm{MeV}$ ). Neutrons in dry air can fly up to $10 \mathrm{~km}$ and cause nuclear reactions when interacting with matter. In this case, the activity will increase significantly.

\section{Conclusion}

There are no international treaties prohibiting or restricting the use of depleted uranium as bullets or shells. There are no political or technological barriers to the wide distribution of such weapons. If it spreads, then soon, any local conflict using the usual small arms available to everyone can lead to radioactive contamination of the area and massive cancerous diseases not only of military personnel performing their duty, but also of civilians. Against the background of foci of cancer and the alienation of territories contaminated with radioactive materials, the bubonic plague, which killed about 60 million people from 1346 to 1353 in Europe, Asia and North Africa, will be only an insignificant episode in the life of human civilization. It is hoped that the world community will finally pay attention to this problem. More and more relevant are the words of Christ: "Put up again thy sword into his place: for all they that take the sword shall perish with the sword." (Matthew 26; 52).

\section{References}

1. Polikanov SM, Uspekhi Fizicheskikh Nauk (1968) 94(1): 43-62.

2. Marakhtanov MK, Okunev VS (2018) The Collective Radioactive Decay of Atomic Nuclei Initiated by an External Mechanical Impact: Science Fiction or a New Class of Physical Processes. Journal of Materials Science Research 7(2) 1-8.

3. Marakhtanov MK, VS Okunev (2018) Physics of the transmutation of stable elements at the collision of macro-objects about high speeds. J Phys Conf Ser 1115. 